\title{
Fast Consensus of Networked Multiagent Systems with Two-Hop Network
}

\author{
Jinfeng Gao, Bin Bu, Lihui Feng, and Minming Gu \\ Department of Automation, Zhejiang Sci-Tech University, Hangzhou 310018, China \\ Correspondence should be addressed to Jinfeng Gao; gaojf163@163.com
}

Received 7 January 2015; Accepted 11 March 2015

Academic Editor: Yun-Bo Zhao

Copyright (C) 2015 Jinfeng Gao et al. This is an open access article distributed under the Creative Commons Attribution License, which permits unrestricted use, distribution, and reproduction in any medium, provided the original work is properly cited.

\begin{abstract}
This paper studies the consensus convergence speed of multiagent systems (MASs) from two aspects including communication topology and the state of agents. Two-hop network is considered in the communication topology. A novel consensus protocol that includes the information of the states motions and their integrals is introduced. And the protocol has much faster convergence speed by choosing some appropriate weight values. The protocol can be applied to distributed control and large-scale systems. A numerical example is presented to illustrate the effectiveness and superiority of the proposed method.
\end{abstract}

\section{Introduction}

Consensus problems for networked multiagent systems (MASs) [1-4] attracted lots of researchers in the last decades, due to their broad applications in many areas such as swarms, flock [5], and multivehicle systems. Some researchers began investigating distributed algorithms for MASs in the early 1990s. A group of simulated robots forms approximations to circles [1]. And different networks are discussed, such as time delay network $[3,6]$, singular network, neural network [7], and random network $[8,9]$. Jadbabaie et al. [10] first present the model of MASs. Vicsek et al. [11] propose the way of agents communication whose information is obtained on the average of their neighbors. The original algorithms of MASs are studied by Olfati-Saber and Murray [12]; they also found the convergence speed is related to algebraic correlation, and some works about connected digraph are mentioned [13]. When creating a MASs network or using a MASs network, some other problems must be solved, such as stabilization, packet dropouts [4, 14], finite-time consensus [15], time delay, quantization, and estimation $[2,16,17]$.

As we know, when network is given, the MASs consensus depends on the protocol. Different protocols have been proposed for various systems. Lin et al. [18] design the loop chase algorithm to make the system get final convergence. As the topology is varied, Jadbabaie et al. [10] consider switch topology to fit varied topology. Now the switch network extends to the random network. Using Markov tools, You [19, $20]$ considers the consensus of the random network which includes continuous and discrete system. Li et al. think about the control protocol of MASs which contain more than one leader [21]. Their main idea is distributed control [22]. Yu et al. study the delay network. They find that if current and delay states of agents are given, system can get final convergence [23].

For improving the convergence speed, fast convergence algorithm is obtained under small world network [24]. Xiao and Boyd [25] consider protocol design as optimization problem; they improve the convergence speed through changing the edge value of topology. However, when the edge value changes, the hardware equipment also changes. Jin and Murray [26] figure it out through changing the way of information transmission. Their main idea is that agents can get not only the information of their neighbors but also the information connecting to these neighbors. And another way to improve convergence speed was proposed [27]; they present a fast convergence algorithm based on PI controller. Through the PI controller, system can get consensus in a tolerant range. 
Can we design a protocol to make convergence speed much faster? Motivated by existing results, a protocol combining the idea about two-hop network with PI controller is proposed in this paper. Two-hop network deals with the information between agents. And PI controller manages every state of agents. With the two advantages, system will converge much faster. Using this protocol, we do not need to change any hardware equipment; just the algorithm makes the convergence situation better. In this paper, consensus analysis is integrated within a formal framework of graph theory, matrix [28, 29], and stability criterion.

This paper is organized as follows. Section 2 raises the question and gives some fundamental issues of MASs. Section 3 illustrates the main results of consensus analysis for first-order and second-order system. Necessary and sufficient conditions for the convergence of the agent's states to a tolerant range value are presented. A numerical example is given in Section 4. The main conclusions are summarized at last.

Notation. The notations are standard. We use a directed graph $G=(V, \varepsilon, A)$ to represent the interaction topology where $V$ is a set of vertices and $\varepsilon \subseteq V^{2}$ is a set of edges. Each edge of the graph is denoted by $\left(v_{i}, v_{j}\right)$ and represents that agent $v_{i}$ has access to the state of agent $v_{j}$. A represents adjacent matrix. The set of neighbors of vertex $v_{i}$ is denoted by $N\left(v_{i}\right)=\left\{v_{j} \in\right.$ $\left.V:\left(v_{i}, v_{j}\right) \in \varepsilon\right\} . L$ represents Laplace matrix. $\widetilde{\varepsilon}$ represents the state of two-hop agent. In this paper, all the topology structures are concerned with undirected graphs.

\section{Preliminaries and Problem Formulation}

For systems modeled by

$$
\dot{x}_{i}=u_{i}, \quad i=1,2, \ldots, N
$$

common protocol is described as

$$
u_{i}(t)=\sum_{j \in N_{i}} a_{i j}\left(x_{j}(t)-x_{i}(t)\right), \quad t \geq 0, i=1,2, \ldots, N,
$$

where $a_{i j}$ is weight of agents' communication. When $\lim _{t \rightarrow \infty}\left\|\left(x_{j}(t)-x_{i}(t)\right)\right\|=0$, system approaches consensus. The necessary and sufficient conditions of consensus of system (1) with protocol (2) are presented [30].

For a multiagent system, two-hop network can make convergence speed become much faster [26]. Agents in twohop network not only get their neighbors' information but also the information by their neighbors. The main idea is that if agents get more information, then system converges much faster. The protocol [26] is

$$
\begin{array}{r}
u_{i}(t)=-\sum_{j \in N_{i}} a_{i j}\left\{x_{i}(t)-x_{j}(t)+\sum_{k \in N_{j}} a_{i j}\left(x_{i}(t)-x_{k}(t)\right)\right\}, \\
t \geq 0, \quad i=1,2, \ldots, N .
\end{array}
$$

Equation (3) can be simplified to

$$
\dot{x}=-L x-\tilde{L} x,
$$

where $u_{i}(t)$ is control input and $x$ is variable of agents state, $x=\left[x_{1}, x_{2}, \ldots, x_{n}\right]^{T}$. Laplacian matrix of MASs is donated by $L ; \widetilde{L}$ is Laplacian matrix of adjacent matrix $\widetilde{A}$. $\widetilde{A}$ is described as

$$
\tilde{a}_{i k}= \begin{cases}\sum_{j \in V} a_{i j} a_{j k}, & \left(v_{i}, v_{k}\right) \in \widetilde{\varepsilon} \\ 0, & \text { other. }\end{cases}
$$

Two-hop network has the information of neighbors and passed neighbors. If the network only contains two nodes, then protocol (3) will degenerate to protocol (2).

Except two-hop network, fast control algorithm based on PI controller also can improve convergence speed [27]; the protocol is

$$
\begin{array}{r}
u_{i}(t)=\sum_{j \in N_{i}} a_{i j} \frac{1}{\theta}\left(\int_{-\theta} x_{j}(t) d t-\int_{-\theta} x_{i}(t) d t\right), \\
t \geq 0, \quad i=1,2, \ldots, N .
\end{array}
$$

$\theta$ represents integral time span which is always the same in this paper. Fast convergence algorithm focuses on state of agents and deals with average value of integral time span. The algorithm can improve dynamic property and convergence speed.

For improving convergence speed, the two methods mentioned above deal with two different aspects of MASs. Two-hop network improves information communication of network topology, and fast convergence algorithm changes agents' state. Combining the advantages of these two methods in this paper, a new protocol to make the convergence speed much faster is proposed. The control protocol is

$$
\begin{aligned}
& \bar{u}_{i}(t)=\alpha u_{i}(t) \\
&-\beta \sum_{j \in N_{i}} a_{i j}\left\{\frac{1}{\theta}\left(\int_{-\theta} x_{i}(t) d t-\int_{-\theta} x_{j}(t) d t\right)\right. \\
&+\sum_{j \in N_{j}} a_{j k} \frac{1}{\theta} \\
&\left.\quad\left(\int_{-\theta} x_{i}(t) d t-\int_{-\theta} x_{j}(t) d t\right)\right\}, \\
& t \geq 0, \quad i=1,2, \ldots, N,
\end{aligned}
$$

where $u_{i}(t)$ is control protocol described in (3), $\alpha$ represents weight value of $u_{i}(t), \beta$ represents weight value of integral gain, and $\theta$ represents integral time span. Now, first-order model and second-order model will be discussed for the convergence speed in Section 3. 


\section{Main Results}

3.1. First-Order Model. The first-order model can be described as (1). Under two-hop network, (7) can be simplified to

$$
\bar{u}=\alpha u-\beta L \frac{1}{\theta} \int_{-\theta} x d t-\beta \widetilde{L} \frac{1}{\theta} \int_{-\theta} x d t .
$$

Theorem 1. Consider MASs (1) with $n$ agents; if topology is connected and undirected, then network systems which are under protocol (8) will converge if and only if $\theta<\pi^{2} / 2 \beta(\lambda+\tilde{\lambda})$, where $(\lambda+\tilde{\lambda})$ represents maximum eigenvalue of Laplacian matrix $(L+\widetilde{L})$.

Proof. Control protocol $u$ described in (1) can get consensus under two-hop network [26], so we just need to prove

$$
\widehat{u}=-\beta L \frac{1}{\theta} \int_{-\theta} x d t-\beta \widetilde{L} \frac{1}{\theta} \int_{-\theta} x d t
$$

Using protocol (9) into first-order model (1), we can get

$$
\dot{x}=-\beta L \frac{1}{\theta} \int_{-\theta} x d t-\beta \widetilde{L} \frac{1}{\theta} \int_{-\theta} x d t
$$

By Laplace transform it becomes

$$
s x-x(0)=-\frac{1}{s \theta} \beta(L+\widetilde{L})\left(x-e^{-s \theta} x\right),
$$

where $x(0)$ is initial value and the closed-loop poles satisfy

$$
\operatorname{det}\left[s I+\frac{1}{s \theta} \beta(L+\widetilde{L})\left(1-e^{-s \theta}\right)\right]=0
$$

Because $(L+\widetilde{L})$ is Laplace matrix and $G$ is connected and undirected graph, one of the poles is $s=0$; then we have

$$
s \prod_{i=2}^{n}\left\{s I+\frac{1}{s \theta} \beta(L+\widetilde{L})\left(1-e^{-s \theta}\right)\right\}=0, \quad i=1,2, \ldots, n .
$$

When $s \neq 0$, we have

$$
1+\frac{1}{s \theta} \beta\left(\lambda_{i}+\tilde{\lambda}_{i}\right)\left(1-e^{-s \theta}\right)=0 .
$$

Because $\left(\lambda_{i}+\tilde{\lambda}_{i}\right) \geq 0$ and $s \leq 0$, its open loop frequency characteristic is as follows:

$$
\begin{gathered}
G(j w) H(j w)=-\frac{1}{w^{2} \theta} \beta\left(\lambda_{i}+\tilde{\lambda}_{i}\right)\left(1-e^{-j w \theta}\right), \\
G|(j w) H(j w)|=-\frac{2}{w^{2} \theta} \beta\left(\lambda_{i}+\tilde{\lambda}_{i}\right)\left|\sin \frac{w \theta}{2}\right|, \\
\arg (G(j w) H(j w))=-\frac{\pi}{2}-\frac{w \theta}{2} .
\end{gathered}
$$

Now, amplitude-frequency characteristic and phasefrequency characteristics are both diminishing. According to Nyquist criterion, when phase angle first arrives to $-\pi$,
$G|(j w) H(j w)|$ in the range of $[-1,0]$, system is stable. Consider

$$
\begin{gathered}
w=\frac{\pi}{\theta}, \\
G|(j w) H(j w)|_{w=\pi / \theta}=\left|\frac{2 \beta \theta(\lambda+\widetilde{\lambda})}{\pi^{2}}\right|<1, \\
\theta<\frac{\pi^{2}}{2 \beta(\lambda+\tilde{\lambda})},
\end{gathered}
$$

where $(\lambda+\tilde{\lambda})$ represents maximum eigenvalue of Laplacian matrix $(L+\widetilde{L})$.

Lemma 2 (see [28]). $G$ is an undirected graph; if $G$ is a connected graph, then the eigenvalues are $0=\lambda_{1}<\lambda_{2} \leq$ $\lambda_{3} \cdots \leq \lambda_{n} ; \lambda_{1}$ equals 0 ; convergence speed is determined by the second minimum eigenvalue $\lambda_{2}$; if $\lambda_{2}$ becomes bigger, then system converges faster.

Theorem 3. Multiagent system (1) under fast convergence algorithm (8), when it is with two-hop network, the convergence speed will become faster if and only if system and protocol have the same parameters and conditions in Theorem 1.

Proof. Consensus algorithm (6) can be simplified to

$$
u(t)=-L \frac{1}{\theta} \int_{-\theta} x d t
$$

The eigenvalues of Laplacian matrix $L$ are $0=\lambda_{1}<\lambda_{2} \leq$ $\lambda_{3} \cdots \leq \lambda_{n}$. When system merges with two-hop network, it can be described as

$$
u(t)=-\beta(L+\widetilde{L}) \frac{1}{\theta} \int_{-\theta} x d t
$$

Eigenvalues of Laplacian matrix $(L+\widetilde{L})$ are $0=\lambda_{1}+\widetilde{\lambda}_{1}<$ $\lambda_{2}+\tilde{\lambda}_{2} \leq \lambda_{3}+\tilde{\lambda}_{3} \cdots \leq \lambda_{n}+\tilde{\lambda}_{n}$. According to Lemma 2, twohop network combined with fast convergence algorithm will improve convergence speed. The proof is thus completed.

If we choose an appropriate weight value in protocol (8) for $\alpha$ and $\beta$, then convergence speed will be satisfied.

3.2. Second-Order Model. The second-order model can be described as

$$
\begin{aligned}
& \dot{m}_{i}=x_{i}, \\
& \dot{x}_{i}=u_{i},
\end{aligned}
$$

where $m, x$ are variables of state and $u$ is controller's input. As a second-order model system, every agent has two states. Its common protocol is

$$
\begin{array}{r}
u_{i}(t)=-\sum_{j \in N} a_{i j}\left\{\beta\left(m_{i}(t)-m_{j}(t)\right)+x_{i}(t)-x_{j}(t)\right\}, \\
t \geq 0, \quad i=1,2, \ldots, N .
\end{array}
$$


In this paper, the protocol combines the fast convergence algorithm with two-hop network, which can be described as

$$
\begin{aligned}
& u_{i}(t)=-r x_{i}(t) \\
& -\sum_{j \in N} a_{i j}\left\{\beta\left(\widehat{m}_{i}(t)-\widehat{m}_{j}(t)\right)\right. \\
& +\beta \sum_{k \in N} w_{j k}\left(\widehat{m}_{i}(t)-\widehat{m}_{k}(t)\right) \\
& +\widehat{x}_{i}(t)-\widehat{x}_{j}(t) \\
& \left.+\sum_{k \in N} w_{j k}\left(\hat{x}_{i}(t)-\widehat{x}_{j}(t)\right)\right\} \text {, } \\
& t \geq 0 \\
& \widehat{m}_{i}(t)=\frac{1}{\theta} \int_{-\theta} m_{i}(t) d t, \quad \widehat{m}_{j}(t)=\frac{1}{\theta} \int_{-\theta} m_{j}(t) d t, \\
& \widehat{x}_{i}(t)=\frac{1}{\theta} \int_{-\theta} x_{i}(t) d t, \quad \widehat{x}_{j}(t)=\frac{1}{\theta} \int_{-\theta} x_{j}(t) d t,
\end{aligned}
$$

where $\beta$ and $r$ are weight values and $\theta$ is integrating range. It can be simplified to

$$
\begin{aligned}
u= & -r x-\beta L \frac{1}{\theta} \int_{-\theta} m d t-\beta \widetilde{L} \frac{1}{\theta} \int_{-\theta} m d t \\
& -L \frac{1}{\theta} \int_{-\theta} x d t, \quad-\widetilde{L} \frac{1}{\theta} \int_{-\theta} x d t .
\end{aligned}
$$

Theorem 4. Consider MASs (19) with $n$ agents; if the communication topology is connected and undirected, then network systems which are under protocol (22) will achieve consensus if and only if $\theta<\pi^{2} / 2(\lambda+\widetilde{\lambda})$, where $(\lambda+\widetilde{\lambda})$ represents maximum eigenvalue of Laplacian matrix $(L+\widetilde{L})$.

Proof. Put control protocol (22) into system (19):

$$
\begin{aligned}
\ddot{m}= & -r \dot{m}-\beta L \frac{1}{\theta} \int_{-\theta} m d t-\beta \widetilde{L} \frac{1}{\theta} \int_{-\theta} m d t \\
& -L \frac{1}{\theta} \int_{-\theta} \dot{m} d t, \quad-\widetilde{L} \frac{1}{\theta} \int_{-\theta} \dot{m} d t .
\end{aligned}
$$

By Laplace transform,

$$
\begin{aligned}
m s^{2}= & -\beta m s-\beta \frac{1}{s \theta}(L+\widetilde{L})\left(m-e^{-s \theta} m\right) \\
& -s \frac{1}{s \theta}(L+\widetilde{L})\left(m-e^{-s \theta} m\right) .
\end{aligned}
$$

Then, the closed-loop poles satisfy

$$
\begin{gathered}
\operatorname{det}\left[s^{2} I+\beta s I+\beta \frac{1}{s \theta}(L+\widetilde{L})\left(1-e^{-s \theta}\right)\right. \\
\left.+s \frac{1}{s \theta}(L+\widetilde{L})\left(1-e^{-s \theta}\right)\right]=0, \\
\operatorname{det}\left[(s I+\beta I)\left(s I+\frac{1-e^{-s \theta}}{s \theta}(L+\widetilde{L})\right)\right]=0 .
\end{gathered}
$$

Because $\beta>0$,

$$
\operatorname{det}\left[s I+\frac{1-e^{-s \theta}}{s \theta}(L+\widetilde{L})\right]=0 \text {. }
$$

Comparing (26) and (12), their constructions are similar. So we can get the same result: when $\theta<\pi^{2} / 2(\lambda+\tilde{\lambda})$, systems converge. $(\lambda+\tilde{\lambda})$ represents maximum eigenvalue of Laplacian matrix $(L+\widetilde{L})$. The proof is thus completed.

Theorem 5. The second-order multiagent system (19) under fast convergence algorithm (22), when it combines with twohop network, the convergence speed will become faster if and only if system and protocol have the same parameters and conditions in Theorem 4.

Proof. Consensus algorithm is

$$
\begin{aligned}
& u_{i}(t)=-r x_{i}(t) \\
&-\sum_{j \in N} a_{i j}\left\{\beta \frac{1}{\theta}\left(\int_{-\theta} m_{j}(t) d t-\int_{-\theta} m_{i}(t) d t\right)\right. \\
&\left.+\frac{1}{\theta}\left(\int_{-\theta} x_{j}(t) d t-\int_{-\theta} x_{i}(t) d t\right)\right\} .
\end{aligned}
$$

It can be simplified to

$$
u=-r x-\beta L \frac{1}{\theta} \int_{-\theta} m d t-L \frac{1}{\theta} \int_{-\theta} x d t
$$

Eigenvalues of Laplacian matrix $L$ are $0=\lambda_{1}<\lambda_{2} \leq$ $\lambda_{3} \cdots \leq \lambda_{n}$. When merging with two-hop network, protocol is described as

$$
u=-r x-\beta(L+\widetilde{L}) \frac{1}{\theta} \int_{-\theta} m d t-(L+\widetilde{L}) \frac{1}{\theta} \int_{-\theta} x d t
$$

Eigenvalues of Laplacian matrix $(L+\widetilde{L})$ are $0=\lambda_{1}+\tilde{\lambda}_{1}<$ $\lambda_{2}+\tilde{\lambda}_{2} \leq \lambda_{3}+\widetilde{\lambda}_{3} \cdots \leq \lambda_{n}+\tilde{\lambda}_{n}$. Except topology, each item is same between (28) and (29), and laplace matrix eigenvalues of topology (29) are bigger than (28). So the system combining the fast convergence algorithm with two-hop network will converge faster. Proof over.

If we choose an appropriate weight value in protocol (22) for $r$ and $\beta$, then convergence speed will be satisfied. We can adjust parameters $r$ and $\beta$ like a PI controller, regard $r$ as proportion parameter, and regard $\beta$ as integration parameter.

\section{Numerical Simulation}

Consider a multiagent system with 4 agents; all agents are first-order model with state $x_{1}, x_{2}, x_{3}, x_{4}$. The initial value is $x=[1 ; 2 ;-1 ;-2]$; Figure 1 shows the network topology. 


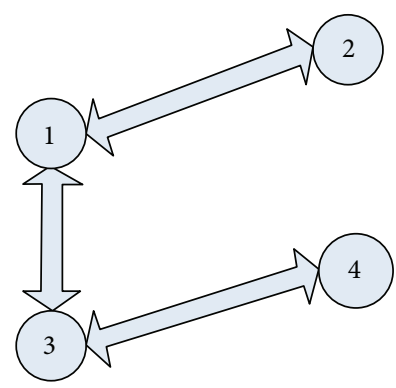

FIGURE 1: Digraph topology with 4 agents.

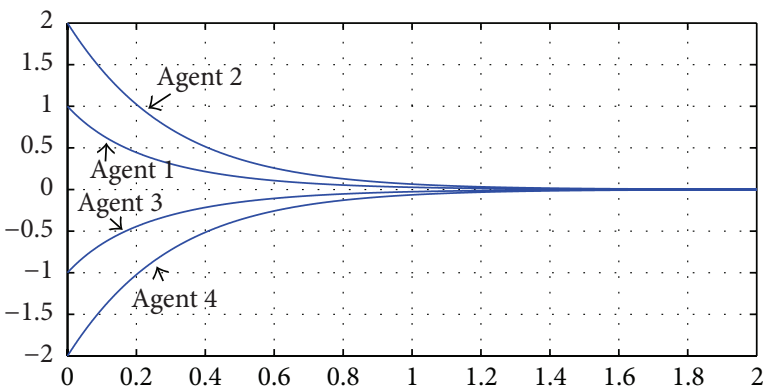

FIGURE 2: System state curves without fast convergence algorithm and two-hop network protocol.

Network topology is undirected graph; the weight value between every edge is 0.5 ; then we get laplace matrix $L$ and $\widetilde{L}$ :

$$
\begin{aligned}
L & =\frac{1}{2}\left(\begin{array}{cccc}
-2 & 1 & 1 & 0 \\
1 & -1 & 0 & 0 \\
1 & 0 & -2 & 1 \\
0 & 0 & 1 & -1
\end{array}\right), \\
\widetilde{L} & =\frac{1}{4}\left(\begin{array}{cccc}
-2 & 1 & 1 & 0 \\
1 & -1 & 0 & 0 \\
1 & 0 & -2 & 1 \\
0 & 0 & 1 & -1
\end{array}\right) .
\end{aligned}
$$

First, simulation result without fast convergence algorithm and two-hop network protocol is shown in Figure 2.

System without fast convergence algorithm and two-hop network protocol converges around $1.40 \mathrm{~s}$.

Secondly, simulation result with fast convergence algorithm without two-hop network protocol is shown in Figure 3.

System with fast convergence algorithm without two-hop network protocol converges around $1.18 \mathrm{~s}$.

Then, simulation result with fast convergence algorithm and two-hop network protocol is shown in Figure $4(\alpha=7$, $\beta=0.3$ ).

System with fast convergence algorithm and two-hop network protocol converges around $0.58 \mathrm{~s}$.

Finally, simulation result with same protocol but different parameter is shown in Figure $5(\alpha=7, \beta=100)$.

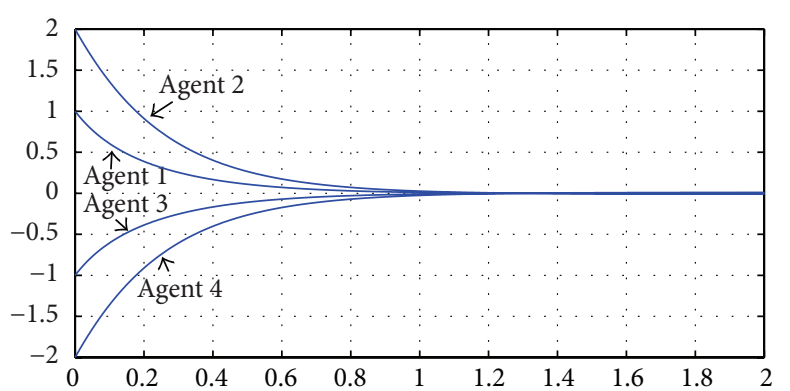

Figure 3: System state curves with fast convergence algorithm without two-hop network protocol.

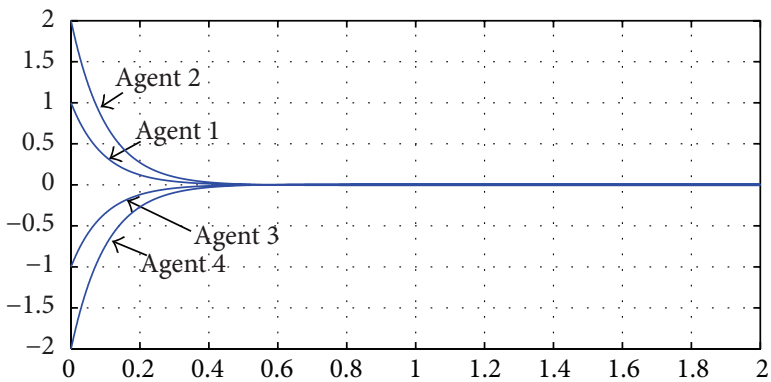

FIGURE 4: System state curves with fast convergence algorithm and two-hop network protocol $\beta=0.3$.

System with same protocol but different parameter will have different consensus performance. In Figure 5 too big parameter $\beta$ gets oscillating curve.

Remark 6. Based on the simulation results showed in Figures $2-4$, state curves with fast convergence and two-hop network converge around $0.58 \mathrm{~s}$ and are better than others. So we can conclude that the proposed algorithm can achieve a much faster convergence speed. The protocol combining fast convergence algorithm with two-hop network protocol is effective. And this paper just improves the protocol. Through this protocol, we do not need to change the hardware equipment; just the protocol makes the performance of system better.

\section{Conclusion}

This paper studies consensus convergence speed of MASs. A control protocol is designed to make system consensus convergence speed much faster. The protocol combines the method of information communication in topology with states of every agent. The necessary and sufficient conditions are obtained based on graph theory and stability theorem. Nevertheless, only first-order model and second-order model are discussed. The protocol cannot be applied to the high order model or multihop network. So the future works will focus on the high order model and multihop network. 


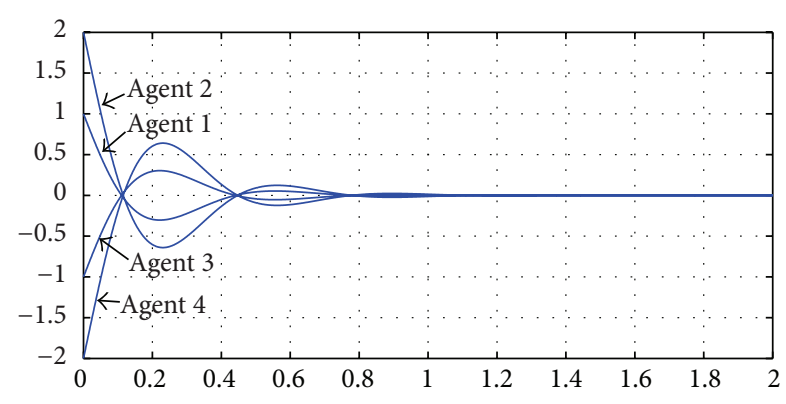

FIgURE 5: System state curves with fast convergence algorithm and two-hop network protocol $\beta=100$.

\section{Conflict of Interests}

The authors declare that there is no conflict of interests regarding the publication of this paper.

\section{Acknowledgments}

This work is supported by National Natural Science Foundation of China under Grant nos. 61374083, 61305135, and 61203177, Science and Technology Department Project of Zhejiang Province under Grant no. 2014C31082, and Natural Science Foundation of Zhejiang Province under Grant no. LQ14F030013, and 521 Talent Project of Zhejiang Sci-Tech University.

\section{References}

[1] K. Sugihara and I. Suzuki, "Distributed motion coordination of multiple mobile robots," in Proceedings of the 5th IEEE International Symposium on Intelligent Control, vol. 1, pp. 138143, IEEE, Philadelphia, Pa, USA, September 1990.

[2] D. E. Marelli and M. Fu, "Distributed weighted least-squares estimation with fast convergence for large-scale systems," Automatica, vol. 51, no. 1, pp. 27-39, 2015.

[3] D. Zhang, W. Cai, and Q.-G. Wang, "Robust non-fragile filtering for networked systems with distributed variable delays," Journal of the Franklin Institute, vol. 351, no. 7, pp. 4009-4022, 2014.

[4] D. Zhang, L. Yu, H. Song, and Q.-G. Wang, "Distributed $H_{\infty}$ filtering for sensor networks with switching topology," International Journal of Systems Science, vol. 44, no. 11, pp. 21042118, 2013.

[5] J. A. Fax and R. M. Murray, "Information flow and cooperative control of vehicle formations," IEEE Transactions on Automatic Control, vol. 49, no. 9, pp. 1465-1476, 2004.

[6] Y. Chen and W. X. Zheng, "Stability analysis of time-delay neural networks subject to stochastic perturbations," IEEE Transactions on Cybernetics, vol. 43, no. 6, pp. 2122-2134, 2013.

[7] Y. Chen and W. X. Zheng, "Stability and L2 performance analysis of stochastic delayed neural networks," IEEE Transactions on Neural Networks, vol. 22, no. 10, pp. 1662-1668, 2011.

[8] Z.-G. Wu, P. Shi, H. Su, and J. Chu, "Passivity analysis for discrete-time stochastic markovian jump neural networks with mixed time delays," IEEE Transactions on Neural Networks, vol. 22, no. 10, pp. 1566-1575, 2011.

[9] Z.-G. Wu, P. Shi, H. Su, and J. Chu, "Stochastic synchronization of markovian jump neural networks with time-varying delay using sampled data," IEEE Transactions on Cybernetics, vol. 43, no. 6, pp. 1796-1806, 2013.

[10] A. Jadbabaie, J. Lin, and A. S. Morse, "Coordination of groups of mobile autonomous agents using nearest neighbor rules," IEEE Transactions on Automatic Control, vol. 48, no. 6, pp. 988-1001, 2003.

[11] T. Vicsek, A. Czirók, E. Ben-Jacob, I. Cohen, and O. Shochet, "Novel type of phase transition in a system of self-driven particles," Physical Review Letters, vol. 75, no. 6, pp. 1226-1229, 1995.

[12] R. Olfati-Saber and R. M. Murray, "Consensus problems in networks of agents with switching topology and time-delays," IEEE Transactions on Automatic Control, vol. 49, no. 9, pp. 15201533, 2004.

[13] W. Ren and R. W. Beard, "Consensus seeking in multiagent systems under dynamically changing interaction topologies," IEEE Transactions on Automatic Control, vol. 50, no. 5, pp. 655661, 2005.

[14] Y. Xu, H. Su, and Y.-J. Pan, “Output feedback stabilization for Markov-based nonuniformly sampled-data networked control systems," Systems and Control Letters, vol. 62, no. 8, pp. 656-663, 2013.

[15] S. H. Yu and X. J. Long, "Finite-time consensus for secondorder multi-agent systems with disturbances by integral sliding mode," Automatica, vol. 54, pp. 158-165, 2015.

[16] R. Lu, Y. Xu, A. Xue, and J. Zheng, "Networked control with state reset and quantized measurements: observer-based case," IEEE Transactions on Industrial Electronics, vol. 60, no. 11, pp. 5206-5213, 2013.

[17] R. Lu, Z. Li, C.-Y. Su, and A. Xue, "Development and learning control of a human limb with a rehabilitation exoskeleton," IEEE Transactions on Industrial Electronics, vol. 61, no. 7, pp. 37763785, 2014.

[18] Z. Lin, M. Broucke, and B. Francis, "Local control strategies for groups of mobile autonomous agents," IEEE Transactions on Automatic Control, vol. 49, no. 4, pp. 622-629, 2004.

[19] K. You, Z. Li, and L. Xie, "Consensus for general multi-agent systems over random graphs," in Proceedings of the 9th IEEE International Conference on Control and Automation (ICCA '11), pp. 830-835, IEEE, December 2011.

[20] K. You, Z. Li, and L. Xie, "Consensus condition for linear multiagent systems over randomly switching topologies," Automatica, vol. 49, no. 10, pp. 3125-3132, 2013.

[21] Z. Li, Z. Duan, W. Ren, and G. Feng, "Containment control of linear multi-agent systems with multiple leaders of bounded inputs using distributed continuous controllers," International Journal of Robust and Nonlinear Control, 2014.

[22] M. P. Fanti, A. M. Mangini, F. Mazzia, and W. Ukovich, "A new class of consensus protocols for agent networks with discrete time dynamics," Automatica, vol. 54, pp. 1-7, 2015.

[23] W. Yu, G. Chen, M. Cao, and W. Ren, "Delay-induced consensus and quasi-consensus in multi-agent dynamical systems," IEEE Transactions on Circuits and Systems, vol. 60, no. 10, pp. 26792687, 2013.

[24] R. Olfati-Saber, "Ultrafast consensus in small-world networks," in Proceedings of the American Control Conference (ACC '05), pp. 2371-2378, IEEE, Portland, Ore, USA, June 2005.

[25] L. Xiao and S. Boyd, "Fast linear iterations for distributed averaging," Systems \& Control Letters, vol. 53, no. 1, pp. 65-78, 2004. 
[26] Z. Jin and R. M. Murray, "Multi-hop relay protocols for fast consensus seeking," in Proceedings of the 45th IEEE Conference on Decision and Control (CDC '06), pp. 1001-1006, San Diego, Calif, USA, December 2006.

[27] Y. Yu, Research on several problems of consensus in multi-agent systems [Ph.D. thesis], Huazhong University of Science and Technology, Wuhan, China, 2010.

[28] R. Horn and C. Johnson, Matrix Analysis, Cambridge University Press, Cambridge, UK, 1985.

[29] B. Mohar and Y. Alavi, "The laplacian spectrum of graphs," in Graph Theory, Combinatorics and Application, vol. 2, pp. 871898, 1991.

[30] Y. Liu and K. M. Passino, "Cohesive behaviors of multiagent systems with information flow constraints," IEEE Transactions on Automatic Control, vol. 51, no. 11, pp. 1734-1748, 2006. 


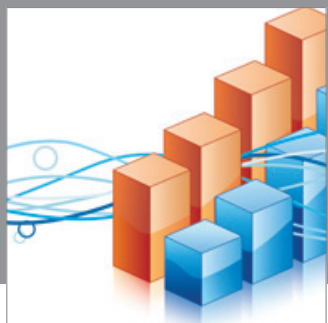

Advances in

Operations Research

mansans

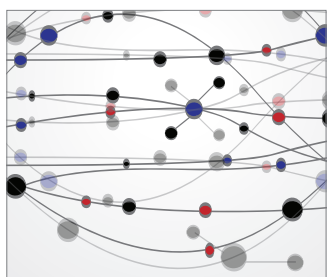

The Scientific World Journal
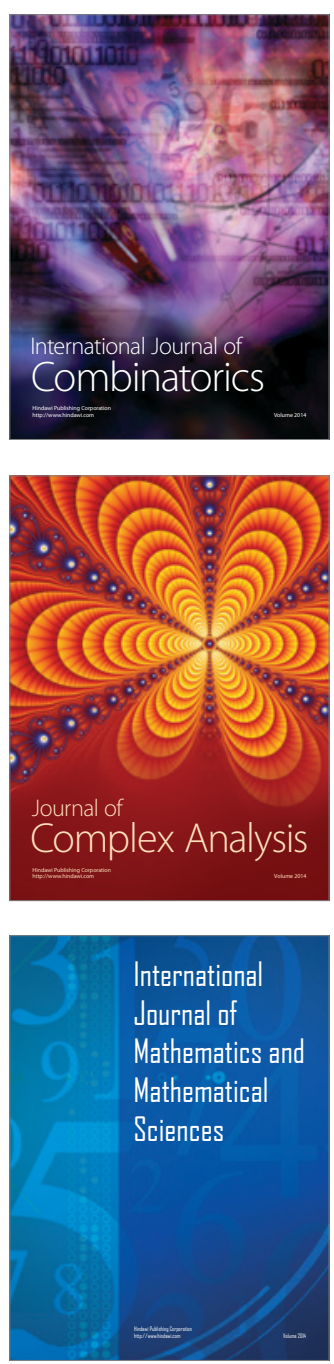
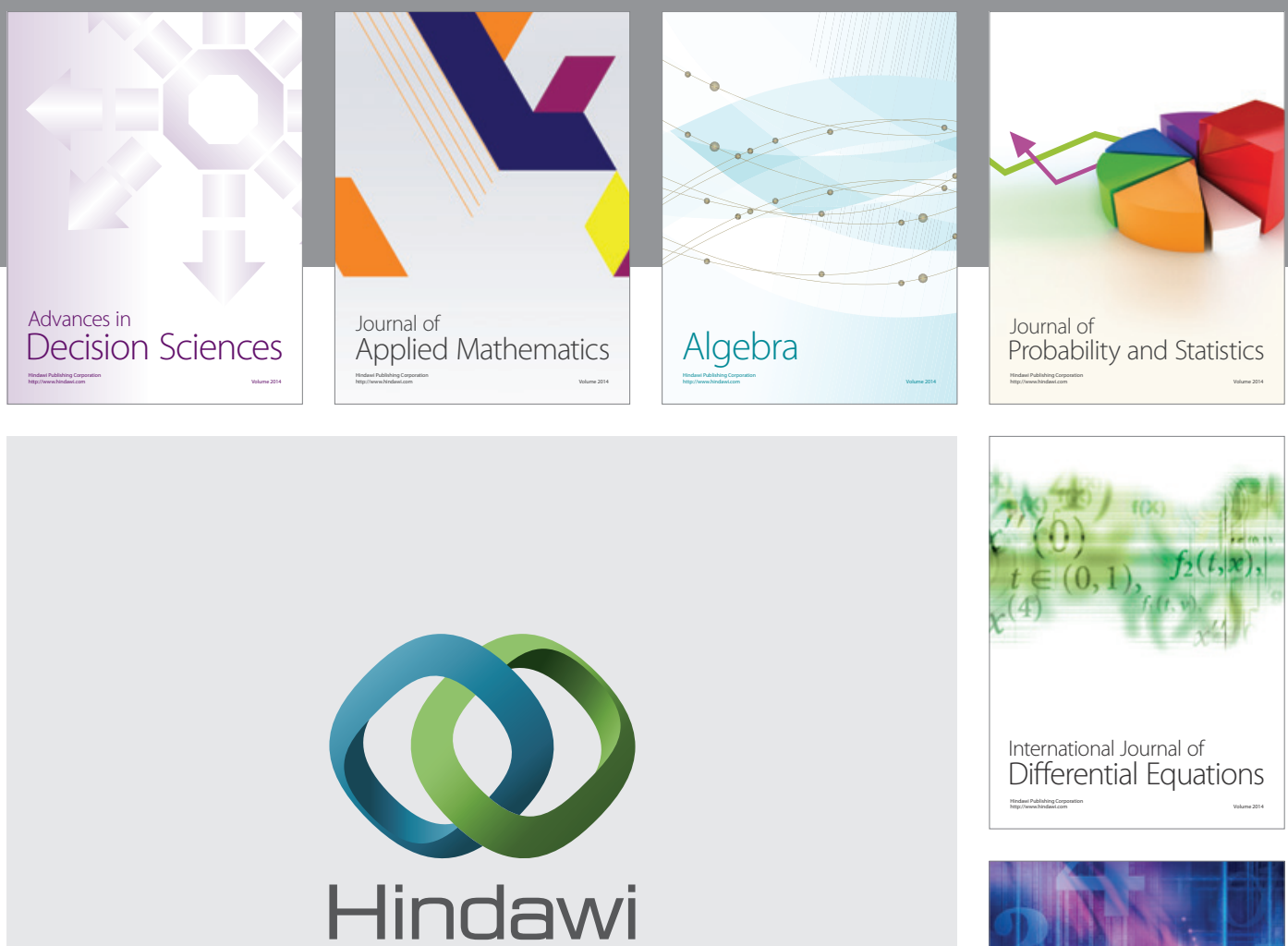

Submit your manuscripts at http://www.hindawi.com
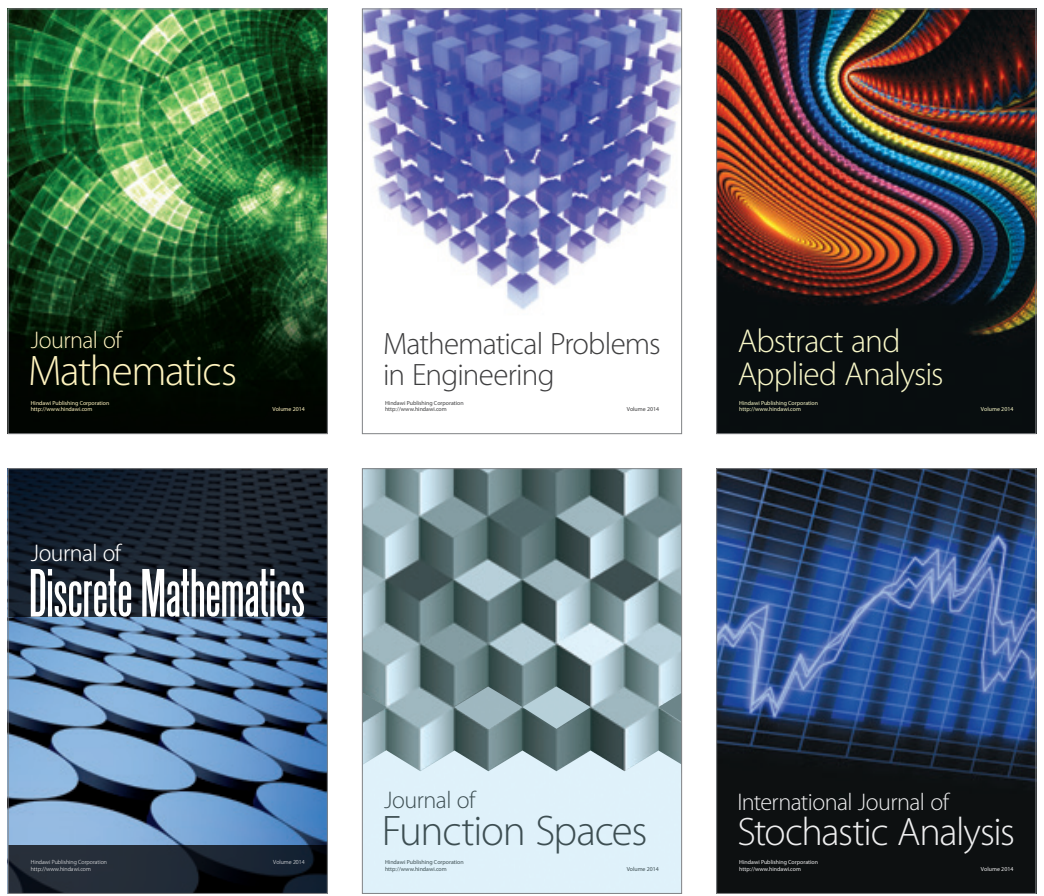

Journal of

Function Spaces

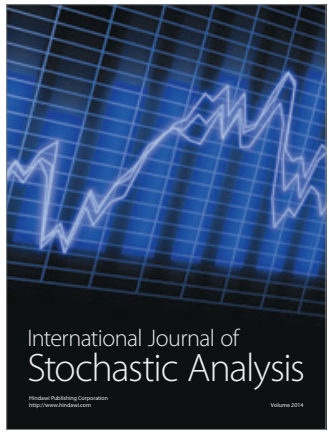

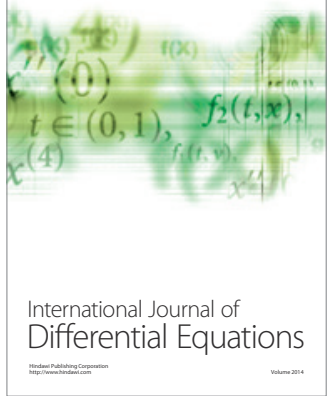
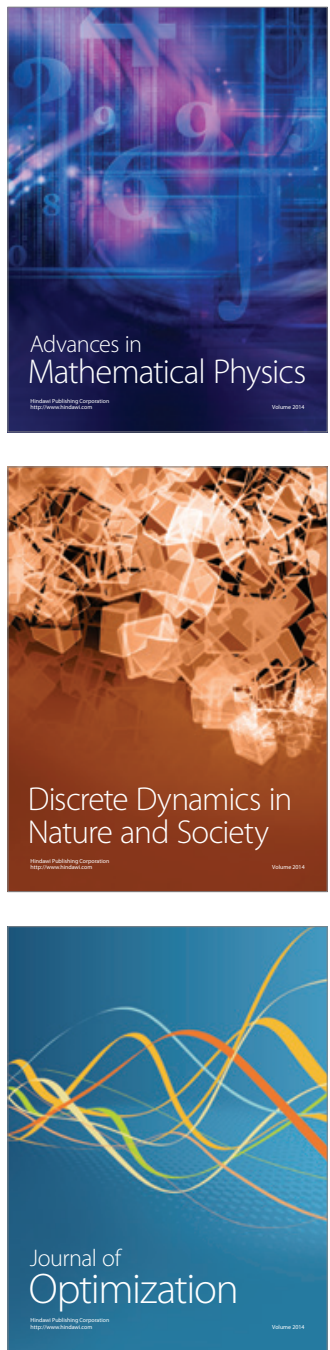